\title{
Necrotizing Fasciitis on the Abdomen, Perineal Area and Thigh after Panperitonitis
}

\author{
Seok Min Yoon ${ }^{1}$, Syeo Young Wee ${ }^{1}$, Hwan Jun Choi \\ ${ }^{1}$ Department of Plastic and Reconstructive Surgery, Soonchunhyang University Gumi Hospital, Gumi; ${ }^{2}$ Department of Plastic and Reconstructive Surgery, \\ Soonchunhyang University Cheonan Hospital, Cheonan, Korea
}

\begin{abstract}
Necrotizing fasciitis (NF) is a rare and rapidly progressive disease involving the skin, subcutaneous tissue, and deep soft tissue. A 76-year-old man presented to the emergency room with melena. Abdominopelvic angiographic computed tomography (CT) revealed a foreign body with calcific density in the ascending colon. Explorative operation was performed, and the foreign body observed on CT was identified to be a chicken bone. Mucosal tearing was found in the ascending colon, and right hemicolectomy was performed. Two days postoperatively, leakage from the anastomosis site was noted and eventually progressed to panperitonitis. One week later, the patient's skin and soft tissue changed to a purplish color, and swelling was observed. As the area gradually increased, a foul odor and purulent discharge were noted. We diagnosed this patient as having NF, and debridement and antibiotic treatment were started. Extendedspectrum $\beta$-lactamases positive Escherichia coli were identified in the excised tissue. After infection subsided, we reconstructed the defect with mesh split-thickness skin graft. The patient recovered without any postoperative complications. NF is an invasive infection that requires prompt diagnosis and treatment. Here, we report successful reconstruction of secondary NF caused by E. coli.
\end{abstract}

Keywords: Fasciitis, necrotizing; Escherichia coli; Intestine perforation; Peritonitis

\section{Introduction}

Necrotizing fasciitis (NF) is a rapidly progressive disease involving the skin and deep soft tissue. Although the incidence is relatively low, reported to be approximately 0.24 to 0.4 out of 100,000 adults, the rate has recently increased, and its mortality rate is high $[1,2]$.

Soft tissue foreign bodies, which often cause superficial or deep tissue infection, are a common reason for emergency room visits [3]. Although NF most commonly occurs due to damage of the external skin surface barrier causing infection, internal organ problems can also lead to infection and NF. Moreira et al. [4], reported perirectal abscess caused by a chicken bone, but extensive NF caused by bowel perforation is still rare. In fact, the incidence of gastrointestinal perforation among foreign body ingestion cases is reported to be less than 1\% [5]. When bowel perforation does occur, it can cause abscess formation due to infection. Even in these cases, however infection is mostly restricted to the abdomen or scrotum, and does not progress to full-fledged NF.

In this study, we report a case of secondary NF after panperitonitis caused by bowel mucosal wall injury due to ingestion of a chicken bone.

\section{Case}

A 76-year-old man with melena visited the emergency room. Abdominopelvic angio-
Case Report

Received: February 2, 2019

Revised: February 23, 2019

Accepted: February 23, 2019

\section{Corresponding author:}

Hwan Jun Choi, M.D., Ph.D.

Department of Plastic and Reconstructive Surgery, Soonchunhyang University Cheonan Hospital, 31 Suncheonhyang 6-gil, Dongnamgu, Cheonan 31151, Korea

Tel: +82-41-570-2195

Fax: +82-41-574-6133

E-mail: 71830@schmc.ac.kr

This work was supported by the

Soonchunhyang University Research Fund.

This is an Open Access article distributed under the terms of the Creative Commons Attribution Non-Commercial License (http://creativecommons.org/licenses/by-nc/4.0/) which permits unrestricted non-commercial use, distribution, and reproduction in any medium, provided the original work is properly cited.

(c) 2019 Korean Wound Management Society 
graphic computed tomography (CT) was performed in the emergency room, and high-density fluid was observed inside the small and large bowel. While this fluid was considered hemorrhage, definite active bleeding was not observed. CT of the ascending colon revealed material with a calcific density (Fig. 1), that was presumed to be a foreign body. The patient was admitted to the department of general surgery for further exploration to identify the cause of melena. A general surgeon performed exploration under general anesthesia, and mucosal tearing was found in the inner wall of the ascending colon due to the foreign body. The foreign body seen on CT was identified as a chicken bone and melena was caused by mucosal tearing of the ascending colon. Bowel viability, reduced due to hemorrhage, was judged to be irreversible, and right hemicolectomy was performed. Two days postoperatively, the patient

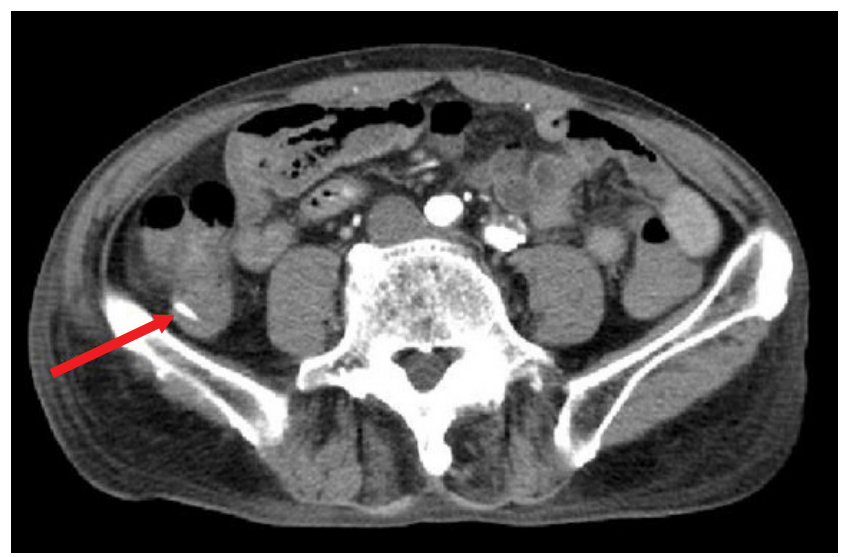

Fig. 1. Initial abdominopelvic angiographic computed tomography finding. A foreign body with calcific density (arrow) was found in the ascending colon.

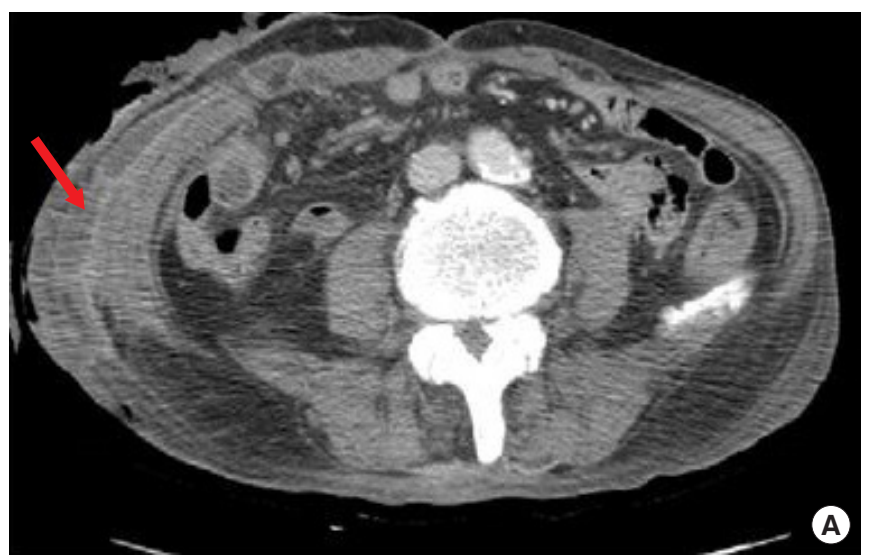

Fig. 2. Computed tomography findings after panperitonitis. (A, B) Collections of fluid containing air bubbles were found in the right flank and thigh (arrow).

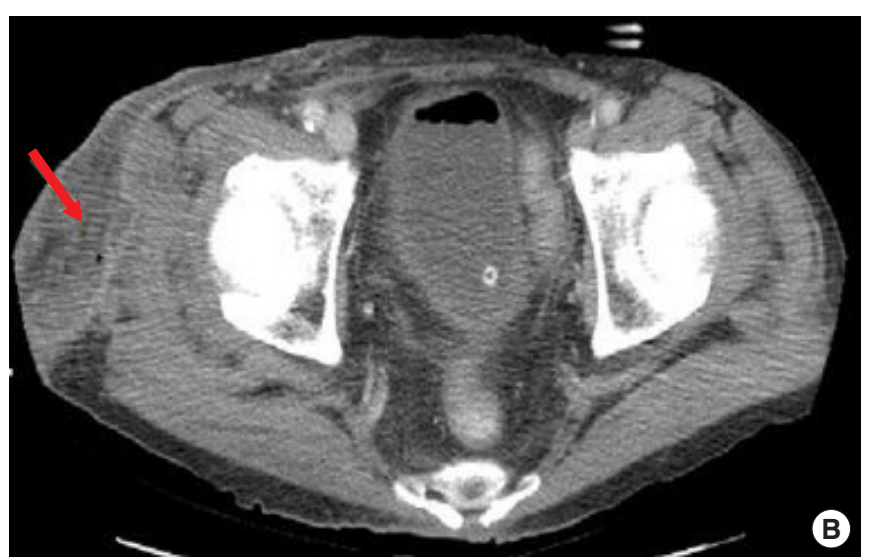

had a fever over $38^{\circ} \mathrm{C}$ and persistent abdominal pain. Bowel distention was also observed. An emergency abdominopelvic CT was performed. Loculated fluid collections containing air bubbles were observed in the right flank and thigh (Fig. 2). Fluid leakage from the anastomosis site was noted and panperitonitis subsequently occurred.

To treat panperitonitis, abdominal exploration was again performed. After sufficient irrigation, segmental resection of unviable ileum was performed. End ileostomy was performed to restore function of the large intestine. After this second surgery, the patient stayed in the intensive care unit for 1 week. After moving to a general room, the patient's abdomen skin and soft tissue turned purplish in color. As the area gradually increased, a foul odor and purulent discharge were present. These skin surface changes had not been detected in the intensive care unit. Epidermolysis progressed in the skin where color change occurred, and it was observed that the soft tissue was involved with partial exposure of muscle fascia. The skin and soft tissue defect area increased not only in the abdomen but also in the perineal area and right thigh.

The patient was referred to the plastic surgery department for wound management. We suspected NF with polymicrobial origin and performed emergent debridement in the operating room. A large amount of necrotic tissue was observed, and the dermis, subcutaneous tissue, and muscle fascia were damaged and displayed purulent discharge. Wound culture was performed on the first debridement. Laboratory test results showed elevations in white blood cell count $\left(26,200 / \mathrm{mm}^{3}\right)$ and C-reactive protein levels $(21.05 \mathrm{mg} / \mathrm{dL}$; normal range $<0.5 \mathrm{mg} / \mathrm{dL})$. Other laboratory parameters (hemoglobin, $13.7 \mathrm{~g} / \mathrm{dL}$; sodium, $133 \mathrm{mmol} / \mathrm{L}$; creatinine, $0.7 \mathrm{mg} / \mathrm{dL}$; and glucose, $135 \mathrm{mg} / \mathrm{dL}$ ) 
were near the normal range.

The culture of excised tissue yielded extended-spectrum beta-lactamases positive Escherichia coli. To eliminate this infection, intravenous antibiotic administration of $1 \mathrm{~g}$ vancomycin daily and $500 \mathrm{mg}$ meropenem twice per day was given for 2

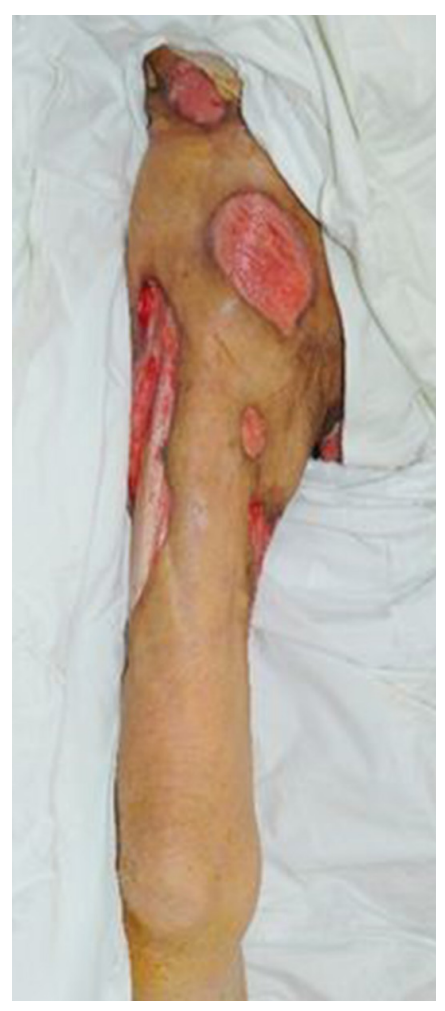

Fig. 3. Photographic finding before skin graft. We performed surgical debridement and negative pressure wound therapy three times per week. Although vastus lateralis muscle fascia was partially exposed, healthy granulation tissue is observed on the raw surfaces as a whole. weeks according to the results of antibiotic sensitivity. Vancomycin infusion was stopped after 2 weeks, and meropenem infusion stopped after 4 weeks.

We performed daily wound irrigation in the operating room, and observed a decrease in the amount of wound discharge after 1 week. The patient's general condition and the intermittent fever also improved. After sign of infection were attenuated, negative pressure wound therapy was performed twice per week for 3 weeks. Granulation tissue formed by 3 weeks of negative pressure wound therapy (Fig. 3). We reconstructed the defect sites with mesh split-thickness skin graft. The skin graft had completely taken 14 days after reconstructive surgery, and the patient recovered without any postoperative complications (Fig. 4).

\section{Discussion}

NF is a rare but serious soft tissue infection. NF can lead to extensive local tissue destruction and, in severe cases, systemic toxicity can lead to multiorgan injury. Patients older than 50 years or who have chronic medical conditions such as diabetes, immunosuppression, obesity and peripheral vascular disease are more likely to progress to NF.

The earliest clinical finding of NF is persistent pain and change of involved skin color, which is due to occlusion of the perforating nutrient vessels and nerve infarction. Pain is induced in the infarctional nerve-dominated skin area. With NF,
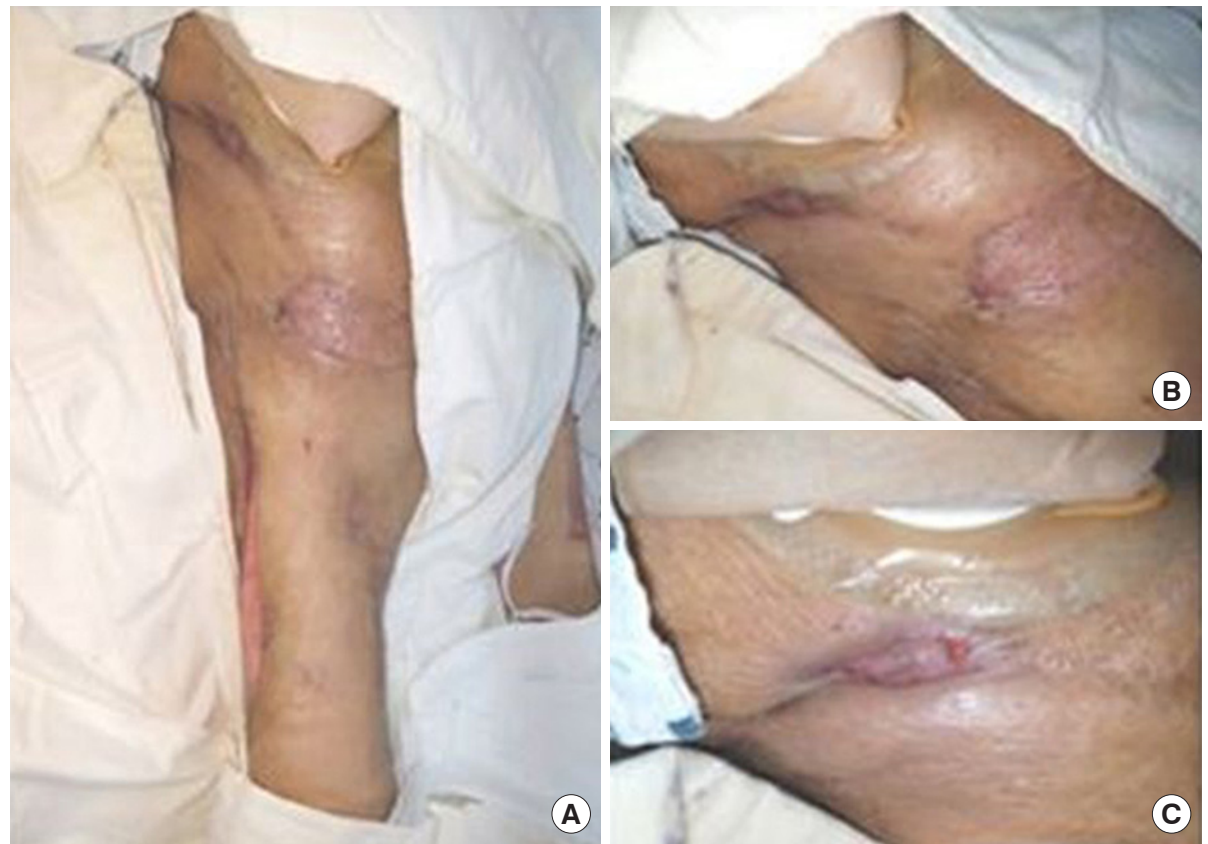

Fig. 4. Postoperative findings. We reconstructed the defect with mesh split thickness skin graft. The patient recovered without any postoperative infection or the other complications. (A, B) Thigh, (C) inguinal crease area. 
the overlying skin is accompanied by redness, vesicles, bullae, necrosis, or crepitus [6].

Simple radiographic imaging, CT, and magnetic resonance imaging can help to diagnose NF which in itself is a surgical emergency. Simple radiographic images are insensitive in the early stages of NF, because findings such as an increase in soft tissue thickness and opacity are similar in both NF and cellulitis. CT images can show abnormal gas distribution along the fascial plane and thickening of the fascial layer along with fluid collection, which findings make it easier to diagnose NF than plain radiographic images. In our case, CT was useful for assessing the extent of soft tissue infection and diagnosing NF.

NF most commonly occurs as infection due to damage of the external skin surface barrier. It can also rarely occur as an infection due to internal organ problems. In our case, abdominal exploration and right hemicolectomy were performed because of mucosal tearing caused by a chicken bone. After abdominal operation, panperitonitis occurred due to anastomosis site leakage. Soft tissue infection then occurred during the treatment of panperitonitis causing NF in the abdomen, perineal area, and thigh. In summary, NF was caused by panperitonitis.

NF is a surgical emergency that should be treated immediately using surgical procedures. In previous studies, if treatment was solely based on antibiotic treatment and support, mortality approached $100 \%$. Surgery is an important treatment for infection source control, the goal of which is to debride all necrotic tissue, leaving and exposing only viable tissue. In a previous study, to treat Fournier's gangrene with fecal contamination, colostomy was performed to resolve contamination, and defect sites were reconstructed by skin graft with vacuum assisted wound therapy [7]. In our case, we performed wound irrigation daily in the operating room. After signs of infection subsided, negative pressure wound therapy was performed twice per week for 3 weeks. After granulation tissue formation, we reconstructed the defect sites with mesh split-thickness skin graft.

Antibiotics can prevent the progression of septic shock when used early. Kumar et al. [8], reported that hypotension caused by septic shock could be reduced if appropriate antibiotics were administrated within the first hours. Broad-spectrum antibiotics should be administrated immediately to combat gram positive, gram negative and anaerobic organisms. If gram negative and positive bacteria are found at the same time, they are usually treated with intravenous carbapenem antibiotics such as meropenem. Recently, the incidence of community acquired methicillin-resistant Staphylococcus aureus (MRSA) NF has increased. Therefore, antibiotics such as vancomycin or linezolid should be used until MRSA has been excluded [9].

Although the incidence is rare, NF should be diagnosed and treated promptly because it progresses rapidly and causes invasive soft tissue infection. While NF most commonly occurs as infection due to damage of the external skin, we should not overlook the fact NF can be caused by internal organ infection such as panperitonitis. In particular, patients with a high risk of infection or changes in skin color should be diagnosed with and treated for NF.

\section{Conflict of interest}

No potential conflicts of interest relevant to this article are reported.

\section{Acknowledgments}

Seok Min Yoon https://orcid.org/0000-0002-9780-814X

Syeo Young Wee https://orcid.org/0000-0002-1787-9715

Hwan Jun Choi https://orcid.org/0000-0002-0752-0389

\section{References}

1. Ellis Simonsen SM, van Orman ER, Hatch BE, et al. Cellulitis incidence in a defined population. Epidemiol Infect 2006; 134:293-9.

2. Salcido RS. Necrotizing fasciitis: reviewing the causes and treatment strategies. Adv Skin Wound Care 2007;20:28893.

3. Yanay O, Vaughan DJ, Diab M, et al. Retained wooden foreign body in a child's thigh complicated by severe necrotizing fasciitis: a case report and discussion of imaging modalities for early diagnosis. Pediatr Emerg Care 2001;17:354-5.

4. Moreira CA, Wongpakdee S, Gennaro AR. A foreign body (chicken bone) in the rectum causing extensive perirectal and scrotal abscess: report of a case. Dis Colon Rectum 1975; 18:407-9.

5. Lo ST, Leung SL, Tang CN. Abdominal wall necrotising fasciitis secondary to fish bone ingestion. J Surg Case Rep 2015; 2015:rjv078.

6. Tan JH, Koh BT, Hong CC, et al. A comparison of necrotising fasciitis in diabetics and non-diabetics: a review of 127 patients. Bone Joint J 2016;98-B:1563-8. 
7. Kim JH, Lee DL, Shin HK, et al. Life-threatening Fournier's gangrene treated by colostomy and vacuum assisted closure. J Wound Management Res 2018;14:120-4.

8. Kumar A, Roberts D, Wood KE, et al. Duration of hypotension before initiation of effective antimicrobial therapy is the critical determinant of survival in human septic shock. Crit
Care Med 2006;34:1589-96.

9. Miller LG, Perdreau-Remington F, Rieg G, et al. Necrotizing fasciitis caused by community-associated methicillin-resistant Staphylococcus aureus in Los Angeles. N Engl J Med 2005;352:1445-53. 